\title{
Categorification at prime roots of unity and hopfological finiteness
}

\author{
You Qi and Joshua Sussan \\ Dedicated to Christian Blanchet on the occasion of his sixtieth birthday
}

\begin{abstract}
We survey some recent results in hopfological algebra and the program of categorification at prime roots of unity. A categorical Jones-Wenzl projector at prime roots of unity is studied, and it is shown that this projector is hopfologically finite in special characteristics, while generically it is infinite.
\end{abstract}

\section{Introduction}

The Witten-Reshetikhin-Turaev (WRT) invariants Wit89, RT90] of links and tangles can be constructed from quantum groups at generic values of $q$. For the purposes of this note, we will only focus on the $\mathfrak{s l}_{2}$-case. When $q$ equals a root of unity, these invariants specialized at $q$ naturally arise, from a quantum physical point of view, as invariants assigned to certain decorated 3-spheres using the 3d-WRT topological quantum field theory (TQFT) $Z_{n}$. Here the $3 \mathrm{~d}$ TQFT $Z_{n}$, parameterized by the order of $q$ (which is related to the level of the TQFT), admits representation theoretical explanations from quantum groups at roots of unity RT90.

The goal of the categorification program envisioned by Crane and Frenkel CF94 was to lift the WRT 3-manifold invariant $Z_{n}\left(M^{3}\right)$, whose values live in the ring of cyclotomic integers $\mathbb{Z}\left[\zeta_{n}\right]$ (see, for instance, BCL14), to a homological invariant. If this hypothetical homological structure were truly a functorial theory, then one should obtain invariants of 4-manifolds with boundaries, which are viewed as cobordisms between 3-manifolds. This would in turn lift $Z_{n}$ to a 4-dimensional theory, as conjectured in CF94.

Strong evidence of the plausibility of Crane and Frenkel's proposal is provided by Khovanov homology Kho00, which assigns a functorial homological invariant to any (framed) tangle. Since this ground-breaking work, there has been significant progress towards categorifying link invariants at generic $q$-values. We refer the reader to the work of Webster Web15. (and the references therein) for more historical accounts, as well as a striking general construction of homological WRT tangle invariants.

In 2004, Khovanov proposed the first steps towards categorification at roots of unity Kho06. He introduced the subject of hopfological algebra, which combines theory of Hopf algebras with homological algebra. The key observation of Kho06 is that, to categorify the ring of cyclotomic integers at a prime root of unity, one could utilize Mayer's homotopy category of $p$-complexes $\mathbb{k}[\partial] /\left(\partial^{p}\right)-\operatorname{gmod}$ May42a, May42b over a field of characteristic $p>0$ :

$$
K_{0}\left(\mathbb{k}[\partial] /\left(\partial^{p}\right)-\underline{\text { gmod }}\right) \cong \mathbb{Z}\left[\zeta_{p}\right]
$$

Mayer's $p$-complexes can be understood as a special case of hopfological algebra, in the sense that it is the hopfological theory of the ground field $\mathbb{k}$ associated with the graded Hopf algebra $H=\mathbb{k}[\partial] /\left(\partial^{p}\right)$, where $\operatorname{char}(\mathbb{k})=p$ and $\operatorname{deg}(\partial)=1$. A characteristic-zero analogue of Mayer's construction, known as $n$-complexes,

2010 Mathematics Subject Classification. Primary 81R50; Secondary 16E20, 16E35.

Key words and phrases. Categorification, hopfological algebra, prime roots of unity.

The authors are very grateful to Mikhail Khovanov for his support and encouragement, as well as sharing with us his insights on the subject. The authors would also like to thank Catharina Stroppel for helpful discussions about categorifying Jones-Wenzl projectors.

J.S. was supported by NSF grant DMS-1407394, PSC-CUNY Award 67144-00 45, and an Alfred P. Sloan Foundation CUNY Junior Faculty Award. 
has been considered by Kapranov Kap96 and Sakaria Sar95. This subject is, roughly speaking, a hopfological theory attached to the Taft algebra $H_{n}$ at an $n$th root of unity. However, due to the lack of a braiding functor on $H_{n}$-mod, it is relatively difficult to implement this theory in the categorification program. It remains an interesting open problem to devise a monoidal categorification of a general cyclotomic integer ring 1 , and apply such a construction in categorification of the WRT 3d TQFT.

In order to apply the "categorical cyclotomic integers" $\mathbb{k}[\partial] /\left(\partial^{p}\right)-\operatorname{gmod}$ to categorification, one needs a good supply of algebra objects in the monoidal category $\mathbb{k}[\partial] /\left(\partial^{p}\right)$-gmod. Such algebra objects arise naturally from $p$-differential graded ( $p$-DG) algebras, an analogue of the usual DG-algebra over graded (super)vector spaces. Some basic technical machinery of hopfological algebra, and in particular, $p$-DG algebra, has been developed in Qi14. The theory parallels that of the usual DG theory, and in fact the usual DG theory is a special case of hopfological algebra for the Hopf superalgebra $\mathbb{k}[d] /\left(d^{2}\right)$. For instance, one has the notion of the abelian category 2 of $p$-DG modules over a $p$-DG algebra $A$, its homotopy category of $p$-DG modules $\mathcal{H}(A)$, and the derived category $\mathcal{D}(A)$ obtained from $\mathcal{H}(A)$ under localization at quasi-isomorphisms. Both categories $\mathcal{H}(A)$ and $\mathcal{D}(A)$ afford categorical module structures over $H$-gmod under tensor product by $p$-complexes. Taking Grothendieck groups in the appropriate sense, the decategorification realizes $K_{0}(\mathcal{D}(A))$ (or $K_{0}\left(\mathcal{H}(A)\right.$ ), but the former is more relevant in our story) as modules over $\mathbb{Z}\left[\zeta_{p}\right]$ :

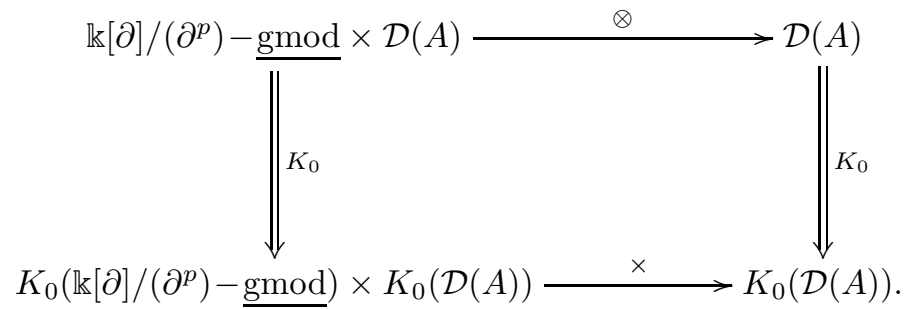

One can also easily check that the usual associativity and distributivity axioms of a module over $\mathbb{Z}\left[\zeta_{p}\right]$ are lifted to the corresponding properties of direct sums and tensor products in the triangulated categories.

In this note we will sketch out what interesting $p$-DG algebras have been investigated in earlier works. A natural way to find nontrivial $p$-DG algebras that may be relevant to quantum topology is to combine hopfological algebra of $p$-differentials with the machineries that have successfully lifted integral quantum structures (for generic $q$ ). It appears to us that, so far, building on the beautiful works of [KL09, KL11, KL10, Lau10, Rou08, Web15 etc., working along this direction seems promising. A summary of what is already known will be carried out in the next few sections, and at the end we will point out one new interesting feature of a hopfological finiteness property and its dependence on the characteristic of the ground field.

In Section 2 we review quantum $\mathfrak{s l}_{2}$ at roots of unity and related structures. The results are by now classical, and are included to fix notation for the rest of the paper.

Starting from Section 3 we recall the definition of a $p$-DG algebra and some basic hopfological properties. Following QS13, we illustrate the definitions with a simple example $A_{n}^{!}$, whose derived category carries a categorical braid group action. Via this example, we hope the reader is convinced that hopfological algebra is indeed connected to quantum topology at roots of unity.

Then we proceed to more representation theoretical aspects of the story. In Section 4 , we review the $p$-DG nilHecke algebra defined by Khovanov and Qi KQ15 used to categorify the lower half of quantum $\mathfrak{s l}_{2}$ at prime roots of unity. This is further extended by Elias and Qi EQ13 to categorify the full small quantum group, following Lauda's Lau10 work on the generic case. A categorification of the BeilinsonLusztig-MacPherson (BLM) form of quantum $\mathfrak{s l}_{2}$ at prime roots of unity has been constructed in EQ15a by equipping the thick calculus of Khovanov-Lauda-Mackaay-Stošić KLMS12 with a compatible $p$-differential.

A categorified Jones-Wenzl projector is constructed in Section 5. When equipped with $p$-differentials, we find some interesting new phenomenon. In certain characteristics, this functor is quasi-isomorphic to a finite complex, although for almost all primes it is an infinite complex unbounded in one direction. This suggests to us that the homology of the colored unknot may be finite only in special prime characteristics. We hope to investigate this phenomenon more carefully in upcoming works.

\footnotetext{
${ }^{1}$ See the recent preprint Mir15] for an interesting new case.

${ }^{2}$ These notions will be recalled in Section 3
} 
Finally, we should mention a recent perspective on variants of WRT TQFTs. The original WRT TQFTs build upon semisimplification of certain tensor subcategories of representation theory of quantum groups at roots of unity. A very recent construction of Blanchet, Costantino, Geer and Patureau-Mirand [BCGPM14] utilizes only projective objects in the representation category to define a non semisimple TQFT. It is an exciting problem to categorify their story at a prime root of unity.

\section{The small quantum group}

2.1. The small quantum group. Let $\zeta_{2 l}$ be a primitive $2 l$ th root of unity. The quantum group $u_{\mathbb{Q}\left[\zeta_{2 l}\right]}\left(\mathfrak{s l}_{2}\right)$, which we will denote simply by $u_{\mathbb{Q}\left[\zeta_{2 l}\right]}$, is the $\mathbb{Q}\left[\zeta_{2 l}\right]$-algebra generated by $E, F, K^{ \pm 1}$ subject to relations:

(1) $K K^{-1}=K^{-1} K=1$,

(2) $K^{ \pm 1} E=\zeta_{2 l}^{ \pm 2} E K^{ \pm 1}, \quad K^{ \pm 1} F=\zeta_{2 l}^{\mp 2} F K^{ \pm 1}$,

(3) $E F-F E=\frac{K-K^{-1}}{\zeta_{2 l}-\zeta_{2 l}^{-1}}$,

(4) $E^{l}=F^{l}=0$.

The quantum group is a Hopf algebra whose comultiplication map

$$
\Delta: u_{\mathbb{Q}\left[\zeta_{2 l}\right]} \longrightarrow u_{\mathbb{Q}\left[\zeta_{2 l}\right]} \otimes_{\mathbb{Q}\left[\zeta_{2 l}\right]} u_{\mathbb{Q}\left[\zeta_{2 l}\right]}
$$

is given on generators by

$$
\Delta(E)=E \otimes 1+K \otimes E, \quad \Delta(F)=1 \otimes F+F \otimes K^{-1}, \quad \Delta\left(K^{ \pm 1}\right)=K^{ \pm 1} \otimes K^{ \pm 1} .
$$

For the purpose of categorification, it is more convenient to use the idempotented quantum group $\dot{u}_{\mathbb{Q}\left[\zeta_{2 l}\right]}\left(\mathfrak{s l}_{2}\right)$, which we will denote simply by $\dot{u}_{\mathbb{Q}\left[\zeta_{2 l}\right]}$. It is a non-unital $\mathbb{Q}\left[\zeta_{2 l}\right]$-algebra generated by $E, F$ and idempotents $1_{\lambda}(\lambda \in \mathbb{Z})$, subject to relations:

(1) $1_{\lambda} 1_{\mu}=\delta_{\lambda, \mu} 1_{\lambda}$,

(2) $E 1_{\lambda}=1_{\lambda+2} E, \quad F 1_{\lambda+2}=1_{\lambda} F$,

(3) $E F 1_{\lambda}-F E 1_{\lambda}=[\lambda] 1_{\lambda}$,

(4) $E^{l}=F^{l}=0$.

Here $[\lambda]=\sum_{i=0}^{|\lambda|-1} \zeta_{2 l}^{1-|\lambda|+2 i}$ is the quantum integer specialized at $\zeta_{2 l}$.

The quantum group $\dot{u}_{\mathbb{Q}\left[\zeta_{2 l}\right]}$ has an integral lattice subalgebra which we now recall. For any integer $n \in\{0,1, \ldots, l-1\}$, let $E^{(n)}=\frac{E^{n}}{[n] !}$, and $F^{(n)}=\frac{F^{n}}{[n] !}$. The elements $E^{(n)}, F^{(n)}(0 \leq n \leq l-1)$, and $1_{\lambda}$ $(\lambda \in \mathbb{Z})$ generate an algebra over the ring of cyclotomic integers $\mathcal{O}_{2 l}=\mathbb{Z}\left[\zeta_{2 l}\right]$. Denote this integral form by $\dot{u}_{\zeta_{2 l}}$.

Now let $l=p$ be prime. Introduce the auxiliary ring

$$
\mathbb{O}_{p}=\mathbb{Z}[q] /\left(\Psi_{p}\left(q^{2}\right)\right),
$$

where $\Psi_{p}(q)$ is the $p$-th cyclotomic polynomial. We can define in a similar fashion an integral form $u_{\mathbb{O}_{p}}$ and its dotted version $\dot{u}_{\mathbb{O}_{p}}$ for the small quantum $\mathfrak{s l}_{2}$ over $\mathbb{O}_{p}$. For more details see [KQ15, Section 3.3]. Let the lower half of $u_{\mathbb{O}_{p}}$ be the subalgebra generated by the $F^{(n)}(0 \leq n \leq p-1)$ and denote it by $u_{\mathbb{O}_{p}}^{-}$. Likewise, write the upper half as $u_{\mathbb{O}_{p}}^{+}$.

Let $V_{n}$ be the unique (up to isomorphism) irreducible module for $\dot{u}_{\mathbb{O}_{p}}$ of rank $n+1$. It has a basis $\left\{v_{0}, v_{1}, \ldots, v_{n}\right\}$ such that

$$
F 1_{\lambda} v_{i}=\delta_{\lambda,-n+2 i}[n-i+1] v_{i-1} \quad E 1_{\lambda} v_{i}=\delta_{\lambda,-n+2 i}[i+1] v_{i+1} .
$$

On $V_{1}^{\otimes n}$ there is an action of $u_{\mathbb{O}_{p}}$ given via the comultiplication map $\Delta$. There is also a commuting action of the braid group that factors through the Hecke algebra. We mostly consider the second lowest weight space which we denote by $V_{1}^{\otimes n}[-n+2]$. This is spanned by vectors $v_{i_{1}} \otimes \cdots \otimes v_{i_{n}}$ where $i_{r} \in\{0,1\}$ and exactly one $i_{r}$ is 1 . More generally $V_{1}^{\otimes n}[-n+2 k]$ is spanned by vectors $v_{i_{1}} \otimes \cdots \otimes v_{i_{n}}$ where $i_{r} \in\{0,1\}$ and $k$ of the $i_{r}$ are 1. It is convenient to use another basis of $V_{1}^{\otimes n}[-n+2]$ which is spanned by vectors $l_{r}$ where

$$
l_{r}= \begin{cases}v_{0}^{\otimes(r-1)} \otimes\left(v_{1} \otimes v_{0}-q v_{0} \otimes v_{1}\right) \otimes v_{0}^{\otimes(n-r-1)} & r \neq n \\ v_{0}^{\otimes(n-1)} \otimes v_{1} & r=n .\end{cases}
$$


2.2. The Temperley-Lieb algebra. The Temperley-Lieb algebra $T L_{n}$ is the $\mathbb{O}_{p}$-algebra generated by elements $U_{i}$ for $i=1, \ldots, n-1$ subject to relations

(1) $U_{i}^{2}=-\left(q+q^{-1}\right) U_{i}$,

(2) $U_{i} U_{j}=U_{j} U_{i}$ for $|i-j|>1$,

(3) $U_{i} U_{j} U_{i}=U_{i}$ for $|i-j|=1$.

Define an operator $\tilde{U}$ on $V_{1}^{\otimes 2}$ by

$$
\begin{aligned}
& \tilde{U}\left(v_{0} \otimes v_{0}\right)=0 \\
& \tilde{U}\left(v_{1} \otimes v_{1}\right)=0 \\
& \tilde{U}\left(v_{0} \otimes v_{1}\right)=v_{1} \otimes v_{0}-q v_{0} \otimes v_{1} \\
& \tilde{U}\left(v_{1} \otimes v_{0}\right)=-q^{-1}\left(v_{1} \otimes v_{0}-q v_{0} \otimes v_{1}\right) .
\end{aligned}
$$

Then there is an action of the Temperley-Lieb algebra on $V_{1}^{\otimes n}$ where the generator $U_{i}: V_{1}^{\otimes n} \rightarrow V_{1}^{\otimes n}$ acts by

$$
U_{i}=\mathrm{Id}^{\otimes(i-1)} \otimes \tilde{U} \otimes \mathrm{Id}^{\otimes(n-i-1)} .
$$

In terms of the basis $\left\{l_{1}, \ldots, l_{n}\right\}$ the action of the Temperley-Lieb algebra is given by

$$
U_{i}\left(l_{j}\right)= \begin{cases}-\left(q+q^{-1}\right) l_{i} & \text { if } i=j \\ l_{i} & \text { if }|i-j|=1 \\ 0 & \text { if }|i-j|>1\end{cases}
$$

2.3. The braid group. The braid group $B_{n}$ is generated by elements $t_{i}$ for $i=1, \ldots, n-1$ subject to the relations that

(1) $t_{i} t_{j}=t_{j} t_{i}$ if $|i-j|>1$

(2) $t_{i} t_{i+1} t_{i}=t_{i+1} t_{i} t_{i+1}$ for $i=1, \ldots, n-2$.

For $i=1, \ldots, n-1$, it will be convenient to introduce the inverse of $t_{i}$ and denote it by $t_{i}^{\prime}$.

Define an operator $\tilde{t}$ on $V_{1}^{\otimes 2}$ by

$$
\begin{aligned}
& \tilde{t}\left(v_{0} \otimes v_{0}\right)=v_{0} \otimes v_{0} \\
& \tilde{t}\left(v_{1} \otimes v_{1}\right)=v_{1} \otimes v_{1} \\
& \tilde{t}\left(v_{0} \otimes v_{1}\right)=q v_{1} \otimes v_{0}+\left(1-q^{2}\right) v_{0} \otimes v_{1} \\
& \tilde{t}\left(v_{1} \otimes v_{0}\right)=q v_{0} \otimes v_{1} .
\end{aligned}
$$

Then there is an action of the braid group generator $t_{i}: V_{1}^{\otimes n} \rightarrow V_{1}^{\otimes n}$ given by

$$
t_{i}=\mathrm{Id}^{\otimes(i-1)} \otimes \tilde{t} \otimes \mathrm{Id}^{\otimes(n-i-1)} .
$$

Define an operator $\tilde{t}^{\prime}$ on $V_{1}^{\otimes 2}$ by

$$
\begin{aligned}
& \tilde{t}^{\prime}\left(v_{0} \otimes v_{0}\right)=v_{0} \otimes v_{0} \\
& \tilde{t}^{\prime}\left(v_{1} \otimes v_{1}\right)=v_{1} \otimes v_{1} \\
& \tilde{t}^{\prime}\left(v_{0} \otimes v_{1}\right)=q^{-1} v_{1} \otimes v_{0} \\
& \tilde{t}^{\prime}\left(v_{1} \otimes v_{0}\right)=\left(1-q^{-2}\right) v_{1} \otimes v_{0}+q^{-1} v_{0} \otimes v_{1} .
\end{aligned}
$$

Then there is an action of the braid group generator $t_{i}^{\prime}: V_{1}^{\otimes n} \rightarrow V_{1}^{\otimes n}$ given by

$$
t_{i}^{\prime}=\mathrm{Id}^{\otimes(i-1)} \otimes \tilde{t}^{\prime} \otimes \mathrm{Id}^{\otimes(n-i-1)} .
$$

It is easy to see that these operators preserve each weight space of $V_{1}^{\otimes n}$ and it is straightforward to check that they do indeed satisfy the braid relations. The action of $B_{n}$ on $V_{1}^{\otimes n}[-n+2]$ is the Burau representation.

In terms of the basis $\left\{l_{1}, \ldots, l_{n}\right\}$ the action of the braid group is given by

$$
t_{i}\left(l_{j}\right)= \begin{cases}-q^{2} l_{i} & \text { if } i=j \\ q l_{i}+l_{j} & \text { if }|i-j|=1 \\ l_{j} & \text { if }|i-j|>1\end{cases}
$$




$$
t_{i}^{\prime}\left(l_{j}\right)= \begin{cases}-q^{-2} l_{i} & \text { if } i=j \\ q^{-1} l_{i}+l_{j} & \text { if }|i-j|=1 \\ l_{j} & \text { if }|i-j|>1 .\end{cases}
$$

2.4. The Jones-Wenzl projector. The Jones-Wenzl projector is the unique linear map

$$
p_{n}: V_{1}^{\otimes n} \rightarrow V_{n} \rightarrow V_{1}^{\otimes n}
$$

which commutes with the action of the quantum group and is idempotent. There are many formulas for this map, usually given in terms of Temperley-Lieb generators. When restricting to weight spaces we could write this map in terms of generators of the quantum group

$$
\frac{1}{\left[\begin{array}{c}
n \\
k
\end{array}\right]} E^{(k)} F^{(k)}: V_{1}^{\otimes n}[-n+2 k] \rightarrow V_{1}^{\otimes n}[-n+2 k] .
$$

For full details see [SS].

Proposition 2.1. The operator $\frac{1}{\left[\begin{array}{l}n \\ k\end{array}\right]} E^{(k)} F^{(k)}: V_{1}^{\otimes n}[-n+2 k] \rightarrow V_{1}^{\otimes n}[-n+2 k]$ is equal to the restriction of the Jones-Wenzl projector $p_{n}[-n+2 k]$.

Proof. One checks that $\frac{1}{\left[\begin{array}{l}n \\ k\end{array}\right]} E^{(k)} F^{(k)}$ is idempotent, commutes with $E$ and $F$ and is annihilated by the elements $U_{i}$ of the Temperley-Lieb algebra. For more details see [SS].

\section{A categorical braid group action at a prime root of unity}

3.1. Elements of $p$-DG algebras. As a matter of notation for the rest of the paper, the undecorated tensor product symbol $\otimes$ will always denote tensor product over the ground field $\mathbb{k}$. We first recall some basic notions.

Definition 3.1. Let $\mathbb{k}$ be a field of positive characteristic $p$. A $p$-DG algebra $A$ over $\mathbb{k}$ is a $\mathbb{Z}$-graded $\mathbb{k}$-algebra equipped with a degree-twd ${ }^{3}$ endomorphism $\partial_{A}$, such that, for any elements $a, b \in A$, we have

$$
\partial_{A}^{p}(a)=0, \quad \partial_{A}(a b)=\partial_{A}(a) b+a \partial_{A}(b) .
$$

Compared with the usual DG case, the lack of the usual sign in the second equation above is because of the fact that the Hopf algebra $\mathbb{k}[\partial] /\left(\partial^{p}\right)$ is a genuine Hopf algebra, not a Hopf super-algebra.

As in the DG case, one has the notion of left and right $p$-DG modules.

Definition 3.2. Let $\left(A, \partial_{A}\right)$ be a $p$-DG algebra. A left $p$-DG module $\left(M, \partial_{M}\right)$ is a $\mathbb{Z}$-graded $A$-module endowed with a degree-two endomorphism $\partial_{M}$, such that, for any elements $a \in A$ and $m \in M$, we have

$$
\partial_{M}^{p}(m)=0, \quad \partial_{M}(a m)=\partial_{A}(a) m+a \partial_{M}(m) .
$$

Similarly, one has the notion of a right $p$-DG module.

It is readily checked that the category of left (right) $p$-DG modules, denoted $A_{\partial}-\bmod \left(A_{\partial}^{o p}-\bmod \right)$, is abelian, with morphisms grading preserving $A$-module maps that also commute with differentials. When no confusion can be caused, we will drop all subscripts in differentials.

Definition 3.3. Let $M$ and $N$ be two $p$-DG modules. A morphism $f: M \longrightarrow N$ in $A_{\partial}-\bmod$ is called null-homotopic if there is an $A$-module map $h$ of degree $2-2 p$ such that

$$
f=\sum_{i=0}^{p-1} \partial_{N}^{i} \circ h \circ \partial_{M}^{p-1-i} .
$$

\footnotetext{
${ }^{3}$ In general one should define the degree of $\partial_{A}$ to be one. We adopt this degree only to match earlier grading conventions in categorification. One may adjust the gradings of the algebras we consider so as to make the degree of $\partial_{A}$ to be one, but we choose not to do so.
} 
It is an easy exercise to check that null-homotopic morphisms form an ideal in $A_{\partial}$-mod. The resulting quotient category, denoted $\mathcal{H}(A)$, is called the homotopy category of left $p$-DG modules over $A$, and it is a triangulated category.

The simplest $p$-DG algebra is the ground field equipped with the trivial differential, whose homotopy category is denoted $\mathbb{k}[\partial] /\left(\partial^{p}\right)$-gmod]. In general, given any $p$-DG algebra $A$, one has a forgetful functor

$$
\text { For : } \mathcal{H}(A) \longrightarrow \mathbb{k}[\partial] /\left(\partial^{p}\right)-\underline{\operatorname{gmod}}
$$

by remembering only the underlying $p$-complex structure up to homotopy of any $p$-DG module over $A$. A morphism between two $p$-DG modules $f: M \longrightarrow N$ (or its image in the homotopy category) is called a quasiisomorphism if For $(f)$ is an isomorphism in $\mathbb{k}[\partial] /\left(\partial^{p}\right)$ - gmod. Denoting the class of quasi-isomorphisms in $\mathcal{H}$ by $\mathcal{Q}$, we define the $p$-DG derived category of $A$ to be

$$
\mathcal{D}(A):=\mathcal{H}(A)\left[\mathcal{Q}^{-1}\right]
$$

the localization of $\mathcal{H}(A)$ at quasi-isomorphisms. By construction, $\mathcal{D}(A)$ is triangulated.

Many constructions in the usual homological algebra of DG-algebras translate over into the $p$-DG context without any trouble. We will see a few illustrations of this phenomenon in what follows.

Generalizing some useful concepts from the usual DG theory Kel06, we make the following definitions.

Definition 3.4. Let $A$ be a $p$-DG algebra, and $K$ be a (left or right) $p$-DG module.

(i) The module $K$ is said to satisfy property $(P)$ if there exists an increasing, possibly infinite, exhaustive $\partial_{K}$-stable filtration $F^{\bullet}$, such that each subquotient $F^{\bullet} / F^{\bullet-1}$ is isomorphic to a direct sum of $p$-DG direct summands of $A$.

(ii) The module $K$ is called a finite cell module, if it satisfies property $(\mathrm{P})$, and as an $A$-module, it is finitely generated (necessarily projective by the property $(\mathrm{P})$ requirement).

Property- $(\mathrm{P})$ modules are the analogues of projective modules in usual homological algebra. For instance, the morphism spaces from a property- $(\mathrm{P})$ module to any $p$-DG module coincide in both the homotopy and derived categories.

It is a theorem Qi14, Theorem 6.6] that there are always sufficiently many property- $(\mathrm{P})$ modules: for any $p$-DG module $M$, there is a surjective quasi-isomorphism

$$
\mathbf{p}(M) \longrightarrow M
$$

of $p$-DG modules, with $\mathbf{p}(M)$ satisfying property $(\mathrm{P})$. We will usually refer to such a property-(P) replacement $\mathbf{p}(M)$ for $M$ as a bar resolution. The proof of its existence is similar to that of the usual (simplicial) bar resolution for DG modules over DG algebras. We omit the proof here, but an example of such a construction will be used in the proof of Theorem 5.5.

In a similar vein, finite cell modules play the role of finite projective modules in usual homological algebra. We give an example of a finite cell module whose construction mimics the notion of "one-sided twisted complexes" for DG modules.

Example 3.5. If $A$ is a $p$-DG algebra, then $A$ itself is finite cell. Now, if $a_{0} \in A$ is an element such that $\partial^{p-1}\left(a_{0}\right)=0$, then one can easily check that the differential rule on the rank-two free module $A x \oplus A y$

$$
\partial(a x):=\partial(a) x+a a_{0} y, \quad \partial(a y):=\partial(a) y
$$

defines the structure of a left $p$-DG module which is finite cell. We will schematically depict this module as

$$
A x \stackrel{a_{0}}{\longrightarrow} A y,
$$

even though $a_{0}$ does not necessarily commute with differentials. One should understand this diagram as a filtered $p$-DG module, with $F^{0}=A y$ and $F^{1}=A x \oplus A y$.

A $p$-DG bimodule ${ }_{A} M_{B}$ over two $p$-DG algebras $A$ and $B$ is a $p$-DG module over $A \otimes B^{\text {op }}$. One has the associated derived tensor product functor

$$
M \otimes_{B}^{\mathbf{L}}(-): \mathcal{D}(B) \longrightarrow \mathcal{D}(A), \quad N \mapsto \mathbf{p}(M) \otimes_{B} N,
$$

where $\mathbf{p}(M)$ is a bar resolution for $M$ as a $p$-DG module over $A \otimes B^{\text {op }}$

One useful fact about such functors is the following theorem, whose proof can be found in Qi14, Section 8].

\footnotetext{
${ }^{4}$ This is usually known as the graded stable category of $\mathbb{k}[\partial] /\left(\partial^{p}\right)$.
} 
Theorem 3.6. Let $f: M_{1} \longrightarrow M_{2}$ be a quasi-isomorphism of $p$-DG bimodules. Then $f$ descends to an isomorphism of the induced derived tensor product functors.

As the last example of adapting the usual DG theory to the present situation, one may define for the triangulated category $\mathcal{D}(A)$ its Grothendieck group $K_{0}(A)$ as usual. What we gain as dividend in the current situation is that, since $\mathcal{D}(A)$ is a "categorical module" over $\mathbb{k}[\partial] /\left(\partial^{p}\right)-\operatorname{gmod}$, the abelian group $K_{0}(A)$ naturally has a module structure over the auxiliary cyclotomic ring at a $p$ th root of unity, which was defined in equation (2.1) of the previous Section:

$$
\mathbb{O}_{p}:=K_{0}\left(\mathbb{k}[\partial] /\left(\partial^{p}\right)-\underline{\operatorname{gmod}}\right) .
$$

This explains the diagram (1.2) in the Introduction.

The Grothendieck groups are the algebraic invariants that we will be interested in for certain $p$-DG algebras in this note. As in the DG case, in order to avoid trivial cancellations one should restrict the class of objects used to define $K_{0}(A)$. It turns out that a good condition is the compactness of a $p$-DG module. However, we will not recall this definition, but refer the reader to Qi14, Section 7] for the details.

3.2. The $p$-DG algebra $A_{n}^{!}$. As an illustration of the general theory discussed above, we now present a $p$-DG algebra that is relevant to quantum topology and easy to define.

Let $\mathbb{k}$ be a field of any characteristic $p \geq 0$ (later we will only focus on the case $p>0$ ). Let $n$ be a natural number greater than or equal to two, and $Q_{n}$ be the following quiver:

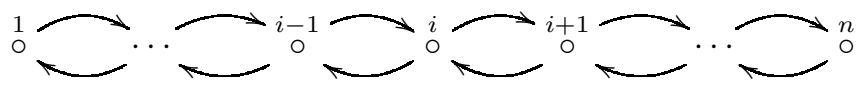

Let $\mathbb{k} Q_{n}$ be the path algebra associated to $Q_{n}$ over the ground field. Following Khovanov-Seidel KS02, we use, for instance, the symbol $(i|j| k)$, where $i, j, k$ are vertices of the quiver $Q_{n}$, to denote the path which starts at a vertex $i$, then goes through $j$ (necessarily $j=i \pm 1$ ) and ends at $k$. The composition of paths is given by

$$
\left(i_{i}\left|i_{2}\right| \cdots \mid i_{r}\right) \cdot\left(j_{1}\left|j_{2}\right| \cdots \mid j_{s}\right)= \begin{cases}\left(i_{i}\left|i_{2}\right| \cdots\left|i_{r}\right| j_{2}|\cdots| j_{s}\right) & \text { if } i_{r}=j_{1}, \\ 0 & \text { otherwise }\end{cases}
$$

where $i_{1}, \ldots, i_{r}$ and $j_{1}, \ldots, j_{s}$ are sequences of neighboring vertices in $Q_{n}$.

Definition 3.7. The algebra $A_{n}^{!}$is the quotient of the path algebra $\mathbb{k} Q_{n}$ by the relations

$$
(1|2| 1)=0 \text { and }(i|i-1| i)=(i|i+1| i) \text { for } i=2, \ldots, n-1 \text {. }
$$

It should be mentioned that $A_{n}^{!}$is isomorphic to the endomorphism algebra of a minimal projective generator of the category $\mathcal{O}_{1, n-1}\left(\mathfrak{g l}_{n}\right)$ considered in BFK99. The sum of categories $\oplus_{i=0}^{n} \mathcal{O}_{i, n-i}\left(\mathfrak{g l}_{n}\right)$ has been used to categorify $V_{1}^{\otimes n}$ in [BFK99]. Moreover, the algebra $A_{n}^{!}$is Koszul, whose quadratic dual is isomorphic to the algebra $A_{n}$ considered in $\mathbf{K S 0 2}$.

This algebra arises naturally from the truncated polynomial algebra $C_{n}:=\mathbb{k}[x] /\left(x^{n}\right)$. For $i=1, \ldots, n$, there are ideal:5

$$
G^{i}=x^{n-i} C_{n}\{i-n\},
$$

where the curly bracket $\{i\}$ stands for a graded module with the grading shifted up by $i$. A routine calculation shows that

$$
A_{n}^{!} \cong \operatorname{END}_{C_{n}}\left(\bigoplus_{i=1}^{n} G^{i}\right)
$$

where the capital $\mathrm{END}_{C_{n}}$ stands for the space of all endomorphisms of a finite-dimensional $C_{n}$-module, not necessarily grading preserving. The path $(i \mid i+1)$ gets sent to the homomorphism $G^{i+1} \rightarrow G^{i}$ which maps $x^{n-i-1}$ to $x^{n-i}$. The path $(i \mid i-1)$ gets sent to the homomorphism $G^{i} \rightarrow G^{i+1}$ which maps $x^{n-i}$ to $x^{n-i}$. The endomorphism algebra inherits a differential determined, for any $\phi \in \operatorname{END}_{C_{n}}\left(\bigoplus_{i=1}^{n} G^{i}\right)$ and $z \in \bigoplus_{i=1}^{n} G^{i}$, by

$$
\partial(\phi)(z)=\partial(\phi(z))-\phi(\partial(z))
$$

\footnotetext{
${ }^{5}$ The ideals $x^{n-i} C_{n}(i=1, \ldots, n)$ are isomorphic to the quotient modules $C /\left(x^{i}\right)$, only as $C$-modules but not as $p$-DG modules. However, the results obtained below will not be affected by using the quotient modules instead: the differentials obtained in different interpretations are conjugate to each other by the involution on $A_{n}^{!}$which reverses arrow directions.
} 
This realization of $A_{n}^{!}$endows the algebra with a $p$-DG structure. On $C_{n}$ there is a differential $\partial$ given by $\partial(x)=x^{2}$. It is clear that each $G^{i}$ is a $p$-DG submodule of $C_{n}$. This gives $A_{n}^{!}$the structure of a $p$-DG algebra where the differential is given by

$$
\partial(i \mid i+1)=(i|i+1| i \mid i+1), \quad \partial(i \mid i-1)=0 .
$$

Since the algebra $A_{n}^{!}$is a finite-dimensional Koszul algebra with $p$-differential acting trivially on the degree zero component, the computation of the Grothendieck group of the derived category of $A_{n}^{!}$reduces to the computation of the Grothendieck without the presence of the differential calculated in Proposition 5.1 See [EQ13, Corollary 2.18] for more details.

Proposition 3.8. There is an isomorphism of $\mathbb{O}_{p}$-modules

$$
K_{0}\left(\mathcal{D}\left(A_{n}^{!}\right)\right) \cong V_{1}^{\otimes n}[-n+2] .
$$

For each $i=1, \ldots, n$ there is a simple left $A_{n}^{!}$-module $L_{i}$ spanned by $1 \in \mathbb{k}$ in degree zero such that $(j) 1=\delta_{i, j} 1$. It is a $p$-DG module with trivial differential. Similarly one may define the simple right $A_{n}^{!}$module ${ }_{i} L$. In the Grothendieck group the image of the module $L_{i}$ gets identified with $l_{i} \in V_{1}^{\otimes n}[-n+2]$ defined earlier.

3.3. A braid group action. For $i=1, \ldots, n-1$ define derived functors $\mathfrak{U}_{i}$ as follows:

$$
\mathfrak{U}_{i}: \mathcal{D}\left(A_{n}^{!}\right) \rightarrow \mathcal{D}\left(A_{n}^{!}\right) \quad \mathfrak{U}_{i}(M)=\left(L_{i} \otimes_{i} L \otimes_{A_{n}^{!}}^{\mathbf{L}} M\right)[-1]\{-1\} .
$$

The following result shows that these functors define a (weak) categorical Temperley-Lieb algebra action on the derived category $\mathcal{D}\left(A_{n}^{!}\right)$.

TheOREm $3.9(\mathbf{Q S 1 3})$. For $i=1, \ldots, n-1$, the functors $\mathfrak{U}_{i}$ are self-biadjoint, and they enjoy the following functor-isomorphism relations.

(i) $\mathfrak{U}_{i} \circ \mathfrak{U}_{i} \cong \mathfrak{U}_{i}[-1]\{-1\} \oplus \mathfrak{U}_{i}[1]\{1\}$,

(ii) $\mathfrak{U}_{i} \circ \mathfrak{U}_{j} \cong \mathfrak{U}_{j} \circ \mathfrak{U}_{i}$ for $|i-j|>1$,

(iii) $\mathfrak{U}_{i} \circ \mathfrak{U}_{j} \circ \mathfrak{U}_{i} \cong \mathfrak{U}_{i}$ for $|i-j|=1$.

These categorical equations can be roughly explained by the fact that the $p$-DG modules $L_{i}$ are spherical objects in the $p$-DG derived category $\mathcal{D}\left(A_{n}^{!}\right)$. Spherical objects in the usual DG case were studied in $\mathbf{A L 1 3}$, ST01, KS02.

To convert spherical objects into braid group generators, we mimic the construction in [KS02 in the $p$-DG context. Consider the following canonical maps:

$$
\begin{gathered}
\epsilon_{1}: L_{i} \otimes{ }_{i} L[-2]\{-2\} \cong L_{i} \otimes \mathbf{R H O M}_{A_{n}^{!}}\left(L_{i}, A_{n}^{!}\right) \stackrel{\lambda_{1}}{\longrightarrow} A_{n}^{!}, \\
\eta_{2}: A_{n}^{!} \stackrel{\lambda_{2}}{\longrightarrow} \mathbf{R H O M}_{\mathbb{k}}\left(\mathbf{R H O M}_{A_{n}^{!}}\left(A_{n}^{!}, L_{i}\right), L_{i}\right) \cong L_{i} \otimes{ }_{i} L .
\end{gathered}
$$

We use them to define filtered $p-D G$ bimodules and derived functors.

Definition 3.10. For each $i \in\{1, \ldots, n-1\}$, we define the functors $\mathfrak{T}_{i}$ and $\mathfrak{T}_{i}^{\prime}$ as follows.

(i) The functor $\mathfrak{T}_{i}: \mathcal{D}\left(A_{n}^{!}\right) \longrightarrow \mathcal{D}\left(A_{n}^{!}\right)$is given by the derived tensor product with the cocone of the bimodule adjunction map (3.9)

$$
A_{n}^{!} \stackrel{\lambda_{2}[-1]}{\longrightarrow} \mathbf{R H O M}_{\mathbb{k}}\left(\mathbf{R H O M}_{A_{n}^{!}}\left(A_{n}^{!}, L_{i}\right), L_{i}\right)[-1] \cong \mathfrak{U}_{i}\{1\}
$$

(ii) The functor $\mathfrak{T}_{i}^{\prime}: \mathcal{D}\left(A_{n}^{!}\right) \longrightarrow \mathcal{D}\left(A_{n}^{!}\right)$is given by the derived tensor product with the cone of the bimodule adjunction map (3.8):

$$
\mathfrak{U}_{i}\{-1\} \cong L_{i} \otimes \mathbf{R H O M}_{A_{n}^{!}}\left(L_{i}, A_{n}^{!}\right)[1] \stackrel{\lambda_{1}[-1]}{\longrightarrow} A_{n}^{!},
$$

TheOREM 3.11. QS13 The functors $\mathfrak{T}_{i}, \mathfrak{T}_{i}^{\prime}$ for $i=1, \ldots, n-1$ satisfy braid relations, i.e., there are isomorphisms of functors

(1) $\mathfrak{T}_{i} \circ \mathfrak{T}_{i}^{\prime} \cong \mathrm{Id} \cong \mathfrak{T}_{i}^{\prime} \circ \mathfrak{T}_{i}$

(2) $\mathfrak{T}_{i} \circ \mathfrak{T}_{j} \cong \mathfrak{T}_{j} \circ \mathfrak{T}_{i}$ if $|i-j|>1$

(3) $\mathfrak{T}_{i} \circ \mathfrak{T}_{i+1} \circ \mathfrak{T}_{i} \cong \mathfrak{T}_{i+1} \circ \mathfrak{T}_{i} \circ \mathfrak{T}_{i+1}$.

The proofs of both Theorems 3.9 and 3.11 are applications of Theorem 3.6 plus explicit comparisons of the bimodules involved. 


\section{A categorification of quantum $\mathfrak{s l}_{2}$ at prime roots of unity}

4.1. The $p$-DG nilHecke category. The ring $C_{n}$ considered in the previous section has a natural topological origin as $C_{n} \cong \mathrm{H}^{*}\left(\mathbb{P}^{n-1}, \mathbb{k}\right)$. Next, we will consider other similarly defined rings from topology, and equip them with $p$-differentials over a field of characteristic $p$.

Let $F l_{n}$ be the flag variety of complete flags in $\mathbb{C}^{n}$. It is well known that the $G L_{n}(\mathbb{C})$-equivariant cohomology of $F l_{n}$ is identified with

$$
\mathrm{H}_{G L_{n}(\mathbb{C})}^{*}\left(F l_{n}, \mathbb{k}\right) \cong \mathrm{H}_{B_{n}}^{*}(\mathrm{pt}, \mathbb{k}) \cong \mathrm{Pol}_{n},
$$

where $B_{n}$ stands for a Borel subgroup of $G L_{n}(\mathbb{C})$, and $\mathrm{Pol}_{n}$ denotes the algebra of polynomial functions on $n$-letters. As such, $\mathrm{Pol}_{n}$ is naturally a module over

$$
\mathrm{H}_{G L_{n}(\mathbb{C})}^{*}(\mathrm{pt}) \cong \Lambda_{n}
$$

The algebra of symmetric functions $\Lambda_{n}$ is nothing but the polynomial algebra on the usual elementary symmetric functions $e_{i}(i=1, \ldots, n)$ on $n$-letters. We define a $p$-DG algebra structure on $\Lambda_{n}$ by setting

$$
\partial\left(e_{i}\right):=e_{1} e_{i}-(i+1) e_{i+1},
$$

with it understood that $e_{n+1}=0$. Write the resulting $p$-DG algebra as $\left(\Lambda_{n}, \partial\right)$.

The nilHecke algebra on n-letters $\mathrm{NH}_{n}$, by definition, is the endomorphism algebra of the module $\mathrm{Pol}_{n}$ over $\Lambda_{n}$ :

$$
\mathrm{NH}_{n} \cong \mathrm{END}_{\Lambda_{n}}\left(\mathrm{Pol}_{n}\right) \text {. }
$$

It is useful to give a generators-and-relations presentation of $\mathrm{NH}_{n}$ as follows. Let $x_{i}(i=1, \ldots, n)$ be the operator of multiplication on $\mathrm{Pol}_{n}$ by the $i$ th variable, and define $\tau_{i}(i=1, \ldots, n-1)$ to be the $i$ th divided difference operator given by

$$
\tau_{i}(f)=\frac{f-{ }^{i} f}{x_{i}-x_{i+1}}
$$

Here $f$ is any polynomial in $\operatorname{Pol}_{n}$, and ${ }^{i} f$ stands for $f$ with the $i$ th and $i+1$ st variables exchanged. A full set of relations satisfied by the generators is given by

$$
\begin{array}{ccc}
x_{i} x_{j} & =x_{j} x_{i}, \quad x_{i} \tau_{j}=\tau_{j} x_{i}(|i-j|>1), & \tau_{i} \tau_{j}=\tau_{j} \tau_{i}(|i-j|>1), \\
\tau_{i}^{2}=0, \quad x_{i} \tau_{i}-\tau_{i} x_{i+1}=1=\tau_{i} x_{i}-x_{i+1} \tau_{i}, & \tau_{i} \tau_{i+1} \tau_{1}=\tau_{i+1} \tau_{i} \tau_{i+1} .
\end{array}
$$

Define

$$
\mathrm{NH}:=\bigoplus_{n \in \mathbb{N}} \mathrm{NH}_{n}-\text { gmod. }
$$

A key property of $\mathrm{NH}$ is that it is a monoidal category, with the monoidal structure coming from a natural inclusion map

$$
\mathrm{NH}_{n} \otimes \mathrm{NH}_{m} \subset \mathrm{NH}_{n+m} .
$$

It is quite surprising to us that, not until the recent work of Khovanov, Lauda and Rouquier KL09, Lau10, Rou08, the following simple relationship between quantum $\mathfrak{s l}_{2}$ and nilHecke algebras, the latter dating back to the work of Bernstein-Gelfand on Schubert calculus, was not stated anywhere in the literature.

Theorem 4.1 ([KL09, Lau10, Rou08]). There is an isomorphism of $\mathbb{Z}\left[q, q^{-1}\right]$-bialgebras

$$
U_{q}^{-}\left(\mathfrak{s l}_{2}\right) \cong K_{0}(\mathrm{NH}),
$$

with the symbols of induction and restriction functors on the right hand side corresponding to the multiplication and comultiplication on $U_{q}^{-}\left(\mathfrak{s l}_{2}\right)$.

To categorify half of the small quantum $\mathfrak{s l}_{2}$, we combine this construction with hopfological algebra of $p$-differentials. To do so, define $\mathrm{Pol}_{n}$ to be the $p$-DG algebra with the $p$-differential $\partial\left(x_{i}\right)=x_{i}^{2}(i=1, \ldots, n)$. Following KQ15, Section 3], define a $p$-DG module structure on the rank-one $\operatorname{Pol}_{n}$ module $\mathcal{P}_{n}$ as follows:

$$
\mathcal{P}_{n}:=\operatorname{Pol}_{n} \cdot v_{0}, \quad \partial\left(v_{0}\right)=-\sum_{i=1}^{n}(n-i) x_{i} v_{0} .
$$

Upon restriction, we regard $\mathcal{P}_{n}$ as a $p$-DG module over $\left(\Lambda_{n}, \partial\right)$. It is shown in KQ15, Section 3] that, up to an isomorphism, this is the unique $p$-DG structure on $\operatorname{Pol}_{n}$ over $\left(\Lambda_{n}, \partial\right)$ that makes the module finite cell 
in all characteristic $p>0$. Furthermore, it also induces a monoidal-structure preserving (see equation (4.6)) differential on the $\mathrm{NH}$ category. A simple computation identifying $\mathrm{NH}_{n} \cong \mathrm{END}_{\Lambda_{n}}\left(\mathcal{P}_{n}\right)$ as $p$-DG algebras yields the following definition.

Definition 4.2. The $p$-DG monoidal category is the direct sum of $p$-DG algebras

$$
(\mathrm{NH}, \partial):=\oplus_{n \in \mathbb{N}}\left(\mathrm{NH}_{n}, \partial\right),
$$

where each $\mathrm{NH}_{n}$ is equipped with the $p$-differential defined on the generators (see equation (4.5)) by

$$
\partial\left(x_{i}\right)=x_{i}^{2}, \quad \partial\left(\tau_{i}\right)=-x_{i} \tau_{i}-\tau_{i} x_{i+1} .
$$

TheOREM 4.3 ([KQ15 $)$. There is an isomorphism of $\mathbb{O}_{p}$-bialgebras

$$
u_{\mathbb{O}_{p}}^{-} \cong K_{0}(\mathcal{D}(\mathrm{NH})) \text {, }
$$

under which $F^{(n)}$ is identified with the symbol $\left[\mathcal{P}_{n}\right]$.

To see that this is indeed the case, we justify the relation $F^{n}=0$ if $n \geq p$. One can readily see that, as a $p$-DG module over $\Lambda_{n}, \mathcal{P}_{n}$ has a $\partial$-stable basis consisting of elements in the set

$$
B_{n}:=\left\{x_{1}^{a_{1}} \ldots x_{n}^{a_{n}} \mid 0 \leq a_{i} \leq n-i\right\} .
$$

The $\mathbb{k}$-span of $B_{n}$ forms an $n$ !-dimensional $p$-complex, which is acyclic whenever $n \geq p$. Therefore, since $\mathrm{NH}_{n} \cong \mathrm{END}_{\Lambda_{n}}\left(\mathcal{P}_{n}\right), \mathrm{NH}_{n}$ is an acyclic $p$-DG algebra whenever $n \geq p$. It follows that $\mathcal{D}\left(\mathrm{NH}_{n}\right) \cong 0$ if $n \geq p$, and this categorical vanishing result lifts the above relation $F^{n}=0(n \geq p)$ for the small quantum group.

4.2. The $p$-DG 2-category $\mathcal{U}$. We seek to "Drinfeld-double" the one-half construction of the categorified small quantum group following Khovanov-Lauda and Rouquier Lau10, KL10, Rou08. To do so for a generic $q$, one takes two copies of the NH-category, and "glue" them together with biadjuntions. More specifically, let us recall the diagrammatic definition of $\mathcal{U}$ introduced by Lauda in Lau10.

Definition 4.4. The 2-category $\mathcal{U}$ is an additive graded $\mathbb{k}$-linear category whose objects $\lambda$ are elements of the weight lattice of $\mathfrak{s l}_{2}$. The 1-morphisms are (direct sums of grading shifts of) composites of the generating 1-morphisms $\mathbb{1}_{\lambda+2} \mathcal{E} \mathbb{1}_{\lambda}$ and $\mathbb{1}_{\lambda} \mathcal{F} \mathbb{1}_{\lambda+2}$, for each $\lambda \in \mathbb{Z}$. Each $\mathbb{1}_{\lambda+2} \mathcal{E} \mathbb{1}_{\lambda}$ will be drawn the same, regardless of the object $\lambda$.

\begin{tabular}{|c|c|c|}
\hline 1-morphism Generator & ${ }^{\lambda+2} \uparrow^{\lambda}$ & ${ }^{\lambda} \bigvee^{\lambda+2}$ \\
\hline Name & $\mathbb{1}_{\lambda+2} \mathcal{E} \mathbb{1}_{\lambda}$ & $\mathbb{1}_{\lambda} \mathcal{F} \mathbb{1}_{\lambda+2}$ \\
\hline
\end{tabular}

The weight of any region in a diagram is determined by the weight of any single region. When no region is labeled, the ambient weight is irrelevant.

The 2-morphisms will be generated by the following pictures.

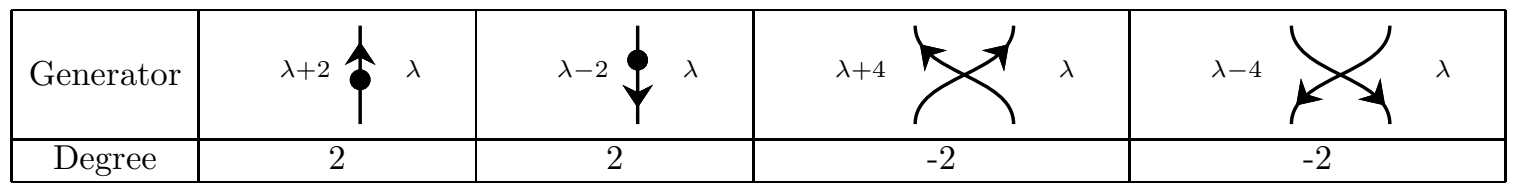

\begin{tabular}{|c|c|c|c|c|}
\hline Generator & $\lambda^{\lambda}$ & $\overbrace{}^{\lambda}$ & $\lambda^{\lambda}$ \\
\hline Degree & $1+\lambda$ & $1-\lambda$ & $1+\lambda$ & $1-\lambda$ \\
\hline
\end{tabular}

We will give a list of relations shortly, after we discuss some notation. For a product of $m$ dots on a single strand, we draw a single dot labeled by $m$. Here is the case when $m=4$.

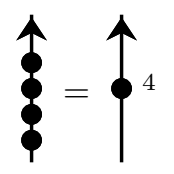


A closed diagram is a diagram without boundary, constructed from the generators above. The simplest non-trivial closed diagram is a bubble, which is a closed diagram without any other closed diagrams inside. Bubbles can be oriented clockwise or counter-clockwise.
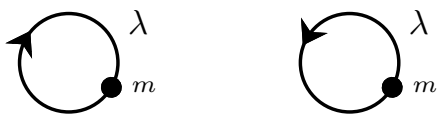

A simple calculation shows that the degree of a bubble with $m$ dots in a region labeled $\lambda$ is $2(m+1-\lambda)$ if the bubble is clockwise, and $2(m+1+\lambda)$ if the bubble is counter-clockwise. Instead of keeping track of the number $m$ of dots a bubble has, it will be much more illustrative to keep track of the degree of the bubble, which is in $2 \mathbb{Z}$. We will use the following shorthand to refer to a bubble of degree $2 k$.


This notation emphasizes the fact that bubbles have a life of their own, independent of their presentation in terms of caps, cups, and dots.

Note that $\lambda$ can be any integer, but $m \geq 0$ because it counts dots. Therefore, we can only construct a clockwise (resp. counter-clockwise) bubble of degree $k$ when $k \geq 1-\lambda$ (resp. $k \geq 1+\lambda$ ). These are called real bubbles. Following Lauda, we also allow bubbles drawn as above with arbitrary $k \in \mathbb{Z}$. Bubbles with $k$ outside of the appropriate range are not yet defined in terms of the generating maps; we call these fake bubbles. One can express any fake bubble in terms of real bubbles (see Remark 4.5).

Now we list the relations. Whenever the region label is omitted, the relation applies to all ambient weights.

(i) Biadjointness relations.
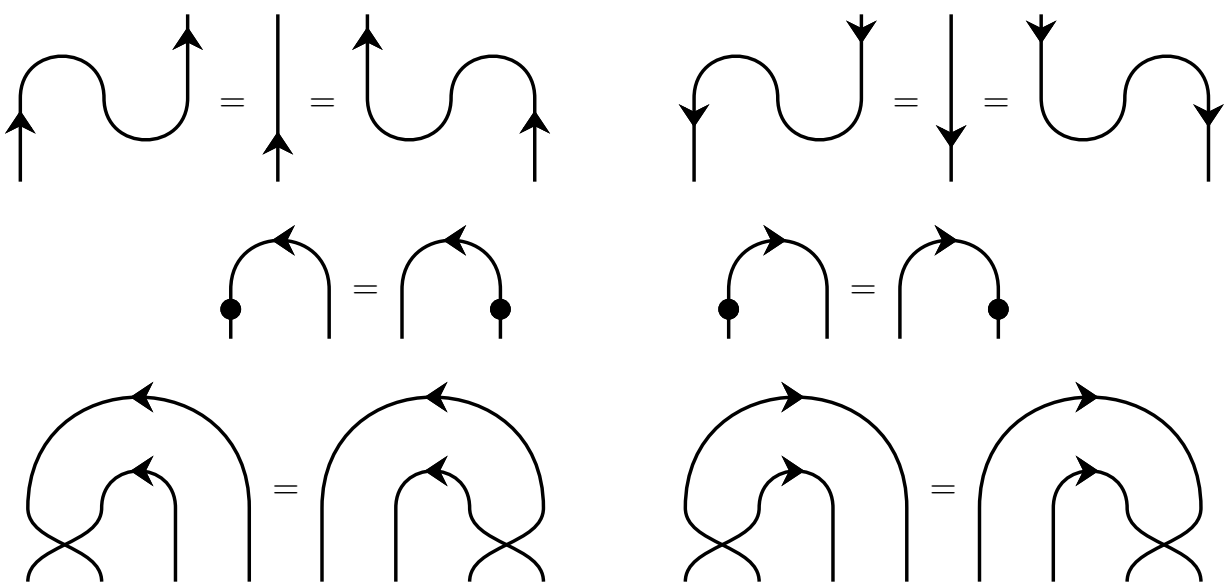

(ii) Positivity and Normalization of bubbles. Positivity states that all bubbles (real or fake) of negative degree should be zero.

$$
\text { ? }=0=0 \text { if } k<0 \text {, }
$$

Normalization states that degree 0 bubbles are equal to the empty diagram (i.e. the identity 2-morphism of the identity 1-morphism).




(iii) NilHecke relations. The upward pointing strands satisfy nilHecke relations as in (4.5). Note that, diagrammatically, far-away commuting elements become isotopy relations and are thus built in by default.
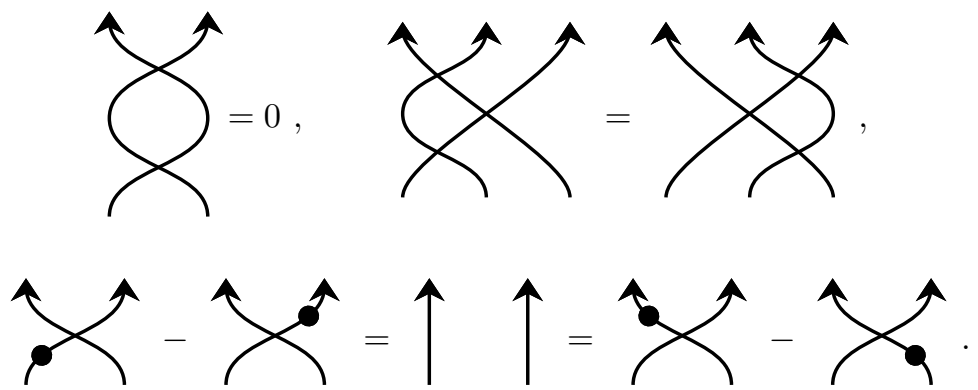

(iv) Reduction to bubbles. The following equalities hold for all $\lambda \in \mathbb{Z}$.
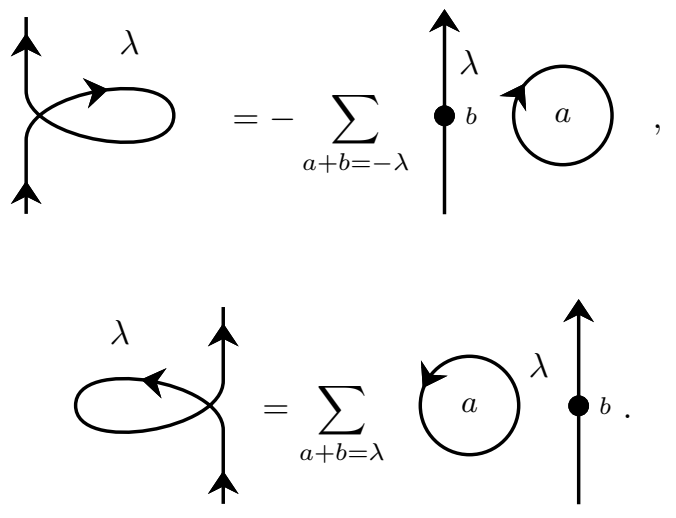

These sums only take values for $a, b \geq 0$. Therefore, when $\lambda \neq 0$, either the right curl or the left curl is zero.

(v) Identity decomposition. The following equations hold for all $\lambda \in \mathbb{Z}$.

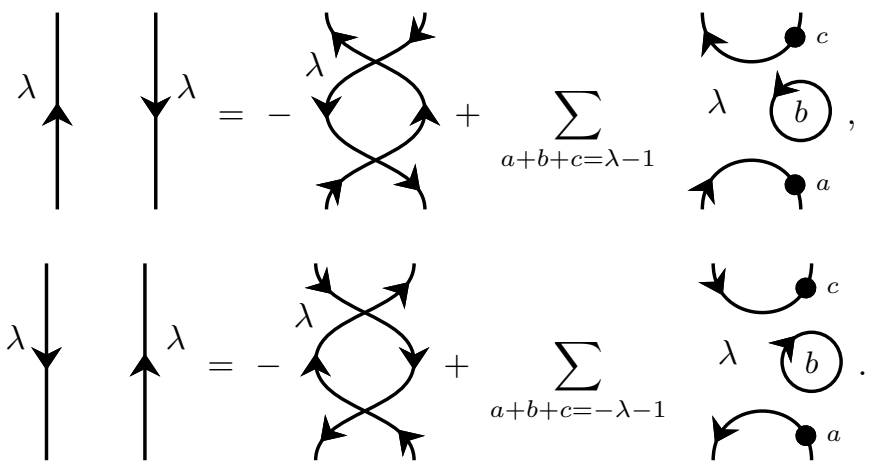

The sum in the first equality vanishes for $\lambda \leq 0$, and the sum in the second equality vanishes for $\lambda \geq 0$.

The terms on the right hand side form a collection of orthogonal idempotents.

REMARK 4.5 (Infinite Grassmannian relations). These relations, which follow from the above defining relations, can be expressed most succinctly in terms of generating functions.

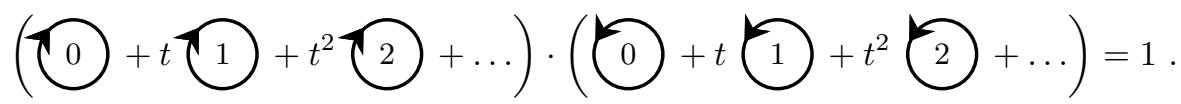

The cohomology ring of the "infinite dimensional Grassmannian" is the ring $\Lambda$ of symmetric functions. Inside this ring, there is an analogous relation $e(t) h(t)=1$, where $e(t)=\sum_{i \geq 0}(-1)^{i} e_{i} t^{i}$ is the total Chern 
class of the tautological bundle, and $h(t)=\sum_{i>0} h_{i} t^{i}$ is the total Chern class of the dual bundle. Lauda has proved that the bubbles in a single region generate an algebra inside $\mathcal{U}$ isomorphic to $\Lambda$.

Looking at the homogeneous component of degree $m$, we have the following equation.

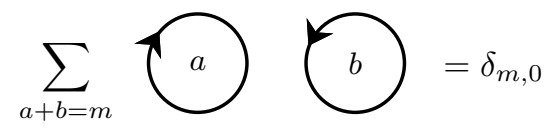

Because of the positivity of bubbles relation, this equation holds true for any $m \in \mathbb{Z}$, and the sum can be taken over all $a, b \in \mathbb{Z}$.

Using these equations one can express all (positive degree) counter-clockwise bubbles in terms of clockwise bubbles, and vice versa. Consequentially, all fake bubbles can be expressed in terms of real bubbles.

Definition 4.6. Let $\partial$ be the derivation defined on the 2-morphism generators of $\mathcal{U}$ as follows.
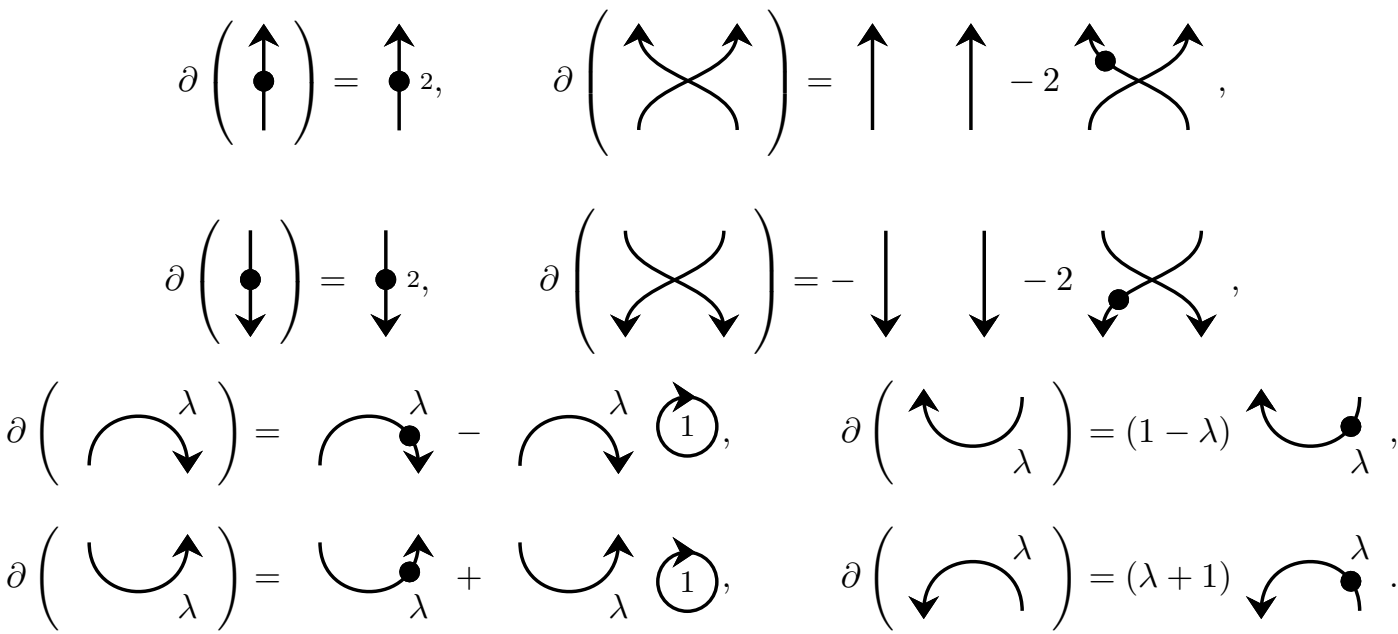

TheOREM 4.7 (EQ13 $)$. There is an isomorphism of $\mathbb{O}_{p}$-algebras

$$
\dot{u}_{\mathbb{O}_{p}} \longrightarrow K_{0}(\mathcal{D}(\mathcal{U}))
$$

sending $1_{\lambda+2} E 1_{\lambda}$ to $\left[\mathbb{1}_{\lambda+2} \mathcal{E} \mathbb{1}_{\lambda}\right]$ and $1_{\lambda} F 1_{\lambda+2}$ to $\left[\mathbb{1}_{\lambda} \mathcal{F} \mathbb{1}_{\lambda+2}\right]$ for any weight $\lambda \in \mathbb{Z}$.

Without the presence of the differential, Khovanov, Lauda, Mackaay and Stošić (KLMS) has given a diagrammatic presentation of the idempotent completion $\dot{\mathcal{U}}$ of $\mathcal{U}$. By construction, $\dot{\mathcal{U}}$ is Morita equivalent to $\mathcal{U}$, and they both categorify quantum $\mathfrak{s l}_{2}$ at generic $q$ values.

The KLMS calculus $\dot{\mathcal{U}}$ has been equipped with a compatible differential derived from $\mathcal{U}$. We will refer the reader to [EQ15a for the details. However, unlike the abelian case, the $p$-DG derived categories are drastically different! There is a functor

$$
\mathcal{J}: \mathcal{H}(\mathcal{U}) \longrightarrow \mathcal{H}(\dot{\mathcal{U}})
$$

which is given by tensor product with $\dot{\mathcal{U}}$ regarded as a $(\dot{\mathcal{U}}, \mathcal{U})$-bimodule with a compatible differential. This functor, not surprisingly, is an equivalence of homotopy categories. However, under localization, it is no longer an equivalence, but instead is a fully-faithful embedding of derived categories:

$$
\mathcal{J}: \mathcal{D}(\mathcal{U}) \longrightarrow \mathcal{D}(\dot{\mathcal{U}})
$$

This derived embedding categorifies the embedding of $\dot{u}_{\mathbb{O}_{p}}$ into the BLM form $\dot{U}_{\mathbb{O}_{p}}$ for quantum $\mathfrak{s l}(2)$, the latter generated by all divided powers $E^{(n)}, F^{(n)}(n \in \mathbb{N})$.

REMARK 4.8. When $p=2$, the constructions in this section can be lifted to characteristic zero, using the usual DG theory. The nilHecke category should then be replaced by the odd nilHecke algebra of [EKL14]. We refer the reader to $\mathbf{E Q 1 5 b}$ for the details. But a natural question still remains: Can one lift the constructions in positive characteristics to characteristic zero? A fundamental problem one needs to tackle is to have a characteristic zero categorification of the ring of cyclotomic integers, and then build nilHecke-like objects in that category. 


\section{A categorification of the Jones-Wenzl projector}

5.1. Categorification at a generic $q$. We present here a special case of a functor categorifying the Jones-Wenzl projector first considered in FSS12 in the context of category $\mathcal{O}$. A further specialization has been studied in greater detail in [SS14, where the Koszul dual functor is computed and shown to be related to the independent constructions of Cooper and Krushkal CK12 and Rozansky Roz14. What follows below is the construction for the category categorifying the second lowest weight space. Details for other weight spaces will appear in $[\mathbf{S S}$.

First we ignore the differential thus categorifying the Jones-Wenzl projector for generic $q$. For ease of notation set

$$
C:=\mathbb{k}[x] /\left(x^{n}\right) \quad A:=A_{n}^{!} .
$$

Proposition 5.1. The Grothendieck groups of finitely generated graded modules over $A$ and $C$ categorify weight spaces of $V_{1}^{\otimes n}$ and $V_{n}$ respectively.

(1) $K_{0}(A-$ gmod $) \cong V_{1}^{\otimes n}[-n+2]$.

(2) $K_{0}(C-$ gmod $) \cong V_{n}[-n+2]$.

Proof. It is easy to see that the projective modules $A(i)$ for $i=1, \ldots, n$ are mutually non-isomorphic and exhaust the list of indecomposable $A$-modules.

The second part is even easier since $C$ is a graded local algebra.

The connection between these categories is given by the Soergel functor and its adjoint. It is easy to see that $\operatorname{END}_{A}(A(n)) \cong C$.

Consider the following two functors. Let

$$
\Pi: A-\operatorname{gmod} \rightarrow C-\operatorname{gmod}
$$

be the exact functor given by

$$
\Pi(M)=(n) A \otimes_{A} M,
$$

which automatically extends to the derived categories. And let

$$
\text { I: } \mathcal{D}^{<}(C \text {-gmod }) \rightarrow \mathcal{D}^{<}(A-\text { gmod })
$$

be the derived functor given by

$$
\mathrm{I}(M)=A(n) \otimes_{C}^{\mathbf{L}} M .
$$

Then define the categorified Jones-Wenzl projector to be

$$
\mathfrak{P}=\mathrm{I} \circ \Pi: \mathcal{D}^{<}(A \text {-gmod }) \rightarrow \mathcal{D}^{<}(A \text {-gmod }) .
$$

In the next result, we construct a projective resolution of $(n) A$ as a graded $(C, A)$-bimodule (recall that unadorned tensor product $\otimes$ is always taken over the ground field).

Proposition 5.2. The following complex of $(C, A)$-bimodules

$$
\cdots \longrightarrow C \otimes(n) A\{2 n+2\} \stackrel{h_{3}}{\longrightarrow} C \otimes(n) A\{2 n\} \stackrel{h_{2}}{\longrightarrow} C \otimes(n) A\{2\} \stackrel{h_{1}}{\longrightarrow} C \otimes(n) A \stackrel{h_{0}}{\longrightarrow}(n) A
$$

is exact, where the differentials are bimodule maps which are defined on the generators by

$$
h_{k}(1 \otimes(n))= \begin{cases}(n) & \text { if } k=0 \\ x \otimes(n)-1 \otimes(n|n-1| n) & \text { if } k=1,3, \ldots \\ \sum_{j=0}^{n-1} x^{j} \otimes(n|n-1| n)^{n-1-j} & \text { if } k=2,4, \ldots\end{cases}
$$

Proof. It is clear that the square of the differential is zero. Thus it suffices to show that the complex is exact.

To do so, notice that, as a left module over $C,(n) A$ decomposes, up to grading shifts, into a direct sum of ideals

$$
(n) A \cong C \oplus x C \oplus \cdots \oplus x^{n-1} C .
$$

Replacing $(n) A$ in each term of the complex by this decomposition, and we are reduced to showing that, for each $i \in\{0,1, \ldots, n-1\}$, the complex

$$
\cdots \longrightarrow C \otimes x^{i} C\{2 n+2\} \stackrel{h_{3}}{\longrightarrow} C \otimes x^{i} C\{2 n\} \stackrel{h_{2}}{\longrightarrow} C \otimes x^{i} C\{2\} \stackrel{h_{1}}{\longrightarrow} C \otimes x^{i} C \stackrel{h_{0}}{\longrightarrow} x^{i} C
$$


is exact. We will first prove it for $i=0$, in this case, the complex is isomorphic to

$$
\cdots \rightarrow \frac{\mathbb{k}[x, y]}{\left(x^{n}, y^{n}\right)}\{2 n+2\} \stackrel{h_{3}}{\longrightarrow} \frac{\mathbb{k}[x, y]}{\left(x^{n}, y^{n}\right)}\{2 n\} \stackrel{h_{2}}{\longrightarrow} \frac{\mathbb{k}[x, y]}{\left(x^{n}, y^{n}\right)}\{2\} \stackrel{h_{1}}{\longrightarrow} \frac{\mathbb{k}[x, y]}{\left(x^{n}, y^{n}\right)} \stackrel{h_{0}}{\longrightarrow} \frac{\mathbb{k}[z]}{\left(z^{n}\right)},
$$

and the boundary maps are now identified with $h_{0}(x)=z, h_{0}(y)=z$, and

$$
h_{k}(1)= \begin{cases}x-y & \text { if } k=1,3, \ldots \\ \sum_{j=0}^{n-1} x^{j} y^{n-1-j} & \text { if } k=2,4, \ldots\end{cases}
$$

The exactness of this complex is clear.

For $i>0$, notice that $x^{i} C \cong C /\left(x^{n-i}\right)$. If one chops off the right most term of equation (5.2), that complex has higher homology groups isomorphic to $\operatorname{Tor}_{C}^{\bullet}\left(C, C /\left(x^{n-i}\right)\right)=0(\bullet>0)$, and the zeroth homology isomorphic to $C /\left(x^{n-i}\right)$. The result follows.

Now tensor the projective resolution in Proposition 5.2 on the left by $A(n)$ over $C$ to get a complex of $(A, A)$-bimodules quasi-isomorphic to the functor $\mathfrak{P}$.

Theorem 5.3. (1) The Jones-Wenzl functor $\mathfrak{P}$ is quasi-isomorphic to the following complex of $(A, A)$-bimodules

$$
\cdots \rightarrow A(n) \otimes(n) A\{2 n+2\} \stackrel{g_{3}}{\longrightarrow} A(n) \otimes(n) A\{2 n\} \stackrel{g_{2}}{\longrightarrow} A(n) \otimes(n) A\{2\} \stackrel{g_{1}}{\longrightarrow} A(n) \otimes(n) A
$$

where

$$
g_{i}= \begin{cases}(n|n-1| n) \otimes 1-1 \otimes(n|n-1| n) & \text { if } \mathrm{i} \text { is odd } \\ \sum_{j=0}^{n-1}(n|n-1| n)^{j} \otimes(n|n-1| n)^{n-1-j} & \text { if } \mathrm{i} \text { is even. }\end{cases}
$$

(2) The functor $\mathfrak{P}$ is idempotent: $\mathfrak{P} \circ \mathfrak{P} \cong \mathfrak{P}$.

(3) The Temperley-Lieb functors $\mathfrak{U}_{i}, i=1, \ldots, n-1$, annhilate the Jones-Wenzl projector:

$$
\mathfrak{U}_{i} \circ \mathfrak{P} \cong 0 \cong \mathfrak{P} \circ \mathfrak{U}_{i} .
$$

Proof. The first statement is clear from the discussion above.

The second statement follows almost directly from the definition of the categorified Jones-Wenzl projector and associativity of (derived) tensor products. The tensor product of the bimodules representing $\mathfrak{P}$ is given by

$$
A(n) \otimes_{C}^{\mathbf{L}}(n) A \otimes_{A} A(n) \otimes_{C}^{\mathbf{L}}(n) A \cong A(n) \otimes_{C}^{\mathbf{L}}(n) A(n) \otimes_{C}^{\mathbf{L}}(n) A \cong A(n) \otimes_{C}^{\mathbf{L}}(n) A .
$$

The result follows.

The third part is a direct consequence of the first part since

$$
{ }_{i} L \otimes{ }_{A}^{\mathbf{L}} A(n) \cong(n) A \otimes_{A}^{\mathbf{L}} L_{i}=0
$$

for $i=1, \ldots, n-1$.

As a 2-representation of Lauda's category this complex may be rewritten as

$$
\left(\cdots \stackrel{f_{4}}{\longrightarrow} \mathcal{E} \mathcal{F}\{2 n+2\} \stackrel{f_{3}}{\longrightarrow} \mathcal{E} \mathcal{F}\{2 n\} \stackrel{f_{2}}{\longrightarrow} \mathcal{E} \mathcal{F}\{2\} \stackrel{f_{1}}{\longrightarrow} \mathcal{E} \mathcal{F}\right)\{n-1\}
$$

where

$$
f_{i}= \begin{cases}x \otimes 1-1 \otimes y & \text { if } i \text { is odd } \\ \sum_{j=0}^{n-1} x^{j} \otimes y^{n-1-j} & \text { if } i \text { is even }\end{cases}
$$

and $x$ and $y$ represent the degree-two endomorphisms of $\mathcal{E}$ and $\mathcal{F}$ respectively. They were diagrammatically represented by a dot on an upward pointing and downward pointing strand respectively in $\mathcal{U}$ (see Section (4).

Corollary 5.4. The equality

$$
[\mathfrak{P}]=\frac{q^{n-1}\left(1-q^{2}\right)}{1-q^{2 n}} E F=\frac{1}{[n]} E F .
$$

holds in the usual Grothendieck group of $\mathcal{U}$. 
5.2. Categorification at a prime root of unity. In the presence of $\partial$ we occasionally expect that the categorified Jones-Wenzl projector is quasi-isomorphic to a finite complex of $(A, A)$-bimodules. On the decategorified level this expectation comes from the observation that for certain values of $p$ the quantity $\frac{1}{n}$ is actually quite simple. For instance, let us write $n-1=k p+r$ with $0 \leq r \leq p-1$. If $p$ divides $n-1$, then we have $[n]=(-1)^{k}$. More generally, if $p$ does not divde $n-1,[n]$ is always a unit in $\mathbb{O}_{p}$. On the level of categories, we will see that, whenever $p$ divides $n-1$, one can construct a finite cell bimodule representing $\mathfrak{P}$. Furthermore, for a fixed $n$, these are precisely all the prime characteristics for which this happens. Indeed we obtain the following theorem:

TheOREm 5.5. Let $n$ be a positive integer, and denote by $A=A_{n}^{!}$and $C=C_{n}$ the $p$-DG algebras which depend on $n$.

(i) If $n-1$ is divisible by $p$, then the $p$-DG bimodule over $(A, A)$ representing $\mathfrak{P}$ is quasi-isomorphic to a finite cell bimodule.

(ii) If $n-1$ is not divisible by $p$, then $\mathfrak{P}$ can not be represented by a finite cell $p$-DG module over $(A, A)$.

Proof of PART (I). To prove the first statement we may explicitly construct a resolution of $\mathfrak{P}$. In this case, $n=k p+1$ for some $k \geq 0$, and the algebra $C=\mathbb{k}[x] /\left(x^{k p+1}\right)$ is quasi-isomorphic to $\mathbb{k}$ since

$$
\mathbb{k}\left(x \rightarrow x^{2} \rightarrow \cdots \rightarrow x^{p}\right) \oplus \mathbb{k}\left(x^{p+1} \rightarrow x^{p+2} \rightarrow \cdots \rightarrow x^{2 p}\right) \oplus \cdots \oplus \mathbb{k}\left(x^{(k-1) p+1} \rightarrow x^{(k-1) p+2} \rightarrow \cdots \rightarrow x^{k p}\right)
$$

is an acyclic ideal inside $C$. Thus, as a $p$-DG bimodule over $(C, A)$, we have a surjective quasi-isomorphism coming from the multiplication map of $C$ on the left module $(n) A$ :

$$
C \otimes(n) A \stackrel{m}{\longrightarrow}(n) A .
$$

It follows that the functor $\mathfrak{P}$ is given by tensor product with the $p$-DG bimodule

$$
A(n) \otimes_{C}^{\mathbf{L}}(n) A \cong A(n) \otimes_{C}(C \otimes(n) A) \cong A(n) \otimes(n) A .
$$

Therefore

$$
\mathfrak{P} \cong(A(n) \otimes(n) A) \otimes_{A}^{\mathbf{L}}(-)=\mathcal{E} \mathcal{F}
$$

It is a bit more difficult to prove the second statement in the Theorem. To do so we will make some preparations.

Lemma 5.6. Let $A$ be a $p$-DG algebra. Suppose

$$
\cdots \stackrel{\phi_{m+4}}{\longrightarrow} P_{m+3} \stackrel{\phi_{m+3}}{\longrightarrow} P_{m+2} \stackrel{\phi_{m+2}}{\longrightarrow} P_{m+1} \stackrel{\phi_{m+1}}{\longrightarrow} P_{m} \longrightarrow 0
$$

is a bounded-from-above exact sequence of $p$-DG modules over $A$. Then the filtered $p$-DG module below, with every other term repeated $(p-1)$-times, is acyclic:

$$
\begin{array}{r}
\cdots \stackrel{\phi_{m+4}}{\longrightarrow} P_{m+3}=\cdots=P_{m+3} \stackrel{\phi_{m+3}}{\longrightarrow} P_{m+2} \longrightarrow \\
\longrightarrow P_{m+1}=\cdots=P_{m+1} \stackrel{\phi_{m+1}}{\longrightarrow} P_{m} \longrightarrow 0 .
\end{array}
$$

Proof. The result reduces easily to the corresponding result for short exact sequences of $p$-DG modules. So let us assume that $P_{k}=0$ when $k \geq m+3$. We will analyze the $p$-complex structure of the filtered $p$-DG module obtained.

As a $p$-complex, it is easy to see that the filtered module 6 has as a subcomplex

$$
0 \longrightarrow \underbrace{\operatorname{Im}\left(\phi_{m+1}\right)=\cdots=\operatorname{Im}\left(\phi_{m+1}\right)}_{(p-1) \text { terms }} \stackrel{\cong}{\longrightarrow} P_{m} \longrightarrow 0
$$

\footnotetext{
${ }^{6}$ Here the filtration on the $p$-DG module is the natural one with respect to the differential: $P_{m}$ is a sub $p$-DG module that is the starting term, and the higher terms in the filtration are obtained by adding more terms to the left of $P_{m}$.
} 
Modulo the subcomplex, the quotient is isomorphic to

$$
0 \longrightarrow P_{m+2} \stackrel{\cong}{\longrightarrow} \underbrace{\operatorname{Ker}\left(\phi_{m+1}\right)=\cdots=\operatorname{Ker}\left(\phi_{m+1}\right)}_{(p-1) \text { terms }} \longrightarrow 0 .
$$

Both complexes are obviously acyclic. The result follows.

ProOF OF PART (II). To prove the statement, we will show that $\mathfrak{P}\left(L_{n}\right)$ has infinite $p$-cohomology. Since $(n) A \otimes_{A} L_{n} \cong \mathbb{k}$, we have $\mathfrak{P}\left(L_{n}\right) \cong A(n) \otimes_{C}^{\mathbf{L}} \mathbb{k}$. We are reduced to finding a property-(P) (Defnition 3.4) resolution for $\mathbb{k}$ as a left $p$-DG module over $C$.

Now we start constructing the resolution. The procedure is an augmented version of the construction of a bar resolution in Qi14, Theorem 6.6], which will be done inductively.

Consider the short exact sequence of $p$-DG modules over $C$

$$
0 \longrightarrow I \longrightarrow C \longrightarrow \mathbb{k} \longrightarrow 0 \text {. }
$$

Here $I$ stands for the ideal generated by $x$, which is a $p$-DG submodule inside $C$.

The surjective multiplication map $C \otimes I \longrightarrow I$ is a map of $p$-DG modules. Denote its kernel by $I_{2}$, and it is easy to see that

$$
I_{2}=\{y \otimes z-1 \otimes y z \mid y, z \in I\} .
$$

As $p$-complexes, $C \otimes I \cong \mathbb{k} \otimes I \oplus I \otimes I$, and thus $I_{2} \cong I \otimes I$. Gluing this map to to the above short exact sequence, we get an exact sequence of left $p$-DG modules

$$
0 \longrightarrow I_{2} \longrightarrow C \otimes I \longrightarrow C \longrightarrow \mathbb{k} \longrightarrow 0 .
$$

Inductively, suppose we have constructed an exact sequence of $p$-DG modules

$$
0 \longrightarrow I_{k-1} \longrightarrow C \otimes I_{k-2} \longrightarrow \cdots \longrightarrow C \otimes I \longrightarrow C \longrightarrow \mathbb{k} \longrightarrow 0
$$

where the arrow between each $C \otimes I_{r} \longrightarrow C \otimes I_{r-1}(r=1, \ldots, k-2)$ is given by the multiplication of $C$ on a $p$-DG module; the kernel $I_{r+1}=\left\{y \otimes w-1 \otimes y w \mid y \in I, w \in I_{r}\right\}$ of the arrow, as a $p$-complex, is isomorphic to $I^{\otimes(r+1)}$.

Then glue to the left most end of the sequence above the $C$ algebra action map

$$
C \otimes I_{k-1} \longrightarrow I_{k-1} \text {. }
$$

by the discussion similar to the $k=2$ case, one sees that the kernel of the map is a $p$-DG submodule inside $C \otimes I_{k-1}$, which can be identified with

$$
I_{k}=\left\{y \otimes w-1 \otimes y w \mid y \in I, w \in I_{k-1}\right\},
$$

whose underlying $p$-complex is isomorphic to $I \otimes I_{k-1} \cong I^{\otimes k}$. This finishes the induction step, and we obtain a bounded-above sequence of $p$-DG modules $\left(I_{1}:=I\right)$.

$$
\cdots \longrightarrow C \otimes I_{k} \longrightarrow C \otimes I_{k-1} \longrightarrow \cdots \longrightarrow C \otimes I_{1} \longrightarrow C \longrightarrow \mathbb{k} \longrightarrow 0 .
$$

Now, applying Lemma 5.6 to the sequence (5.8), we obtain a desired filtered module which satisfies property (P) (Definition 3.4) and is quasi-isomorphic to $\mathbb{k}$ :

$$
\begin{array}{r}
\cdots \stackrel{m}{\longrightarrow} C \otimes I_{3}=\cdots \otimes I_{3} \stackrel{m}{\longrightarrow} C \otimes I_{2} \longrightarrow \\
\longrightarrow C \otimes I_{1}=\cdots \otimes I_{1} \stackrel{m}{\longrightarrow} C \longrightarrow 0,
\end{array}
$$

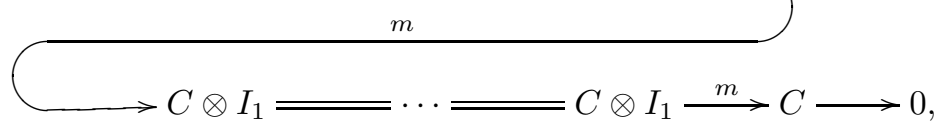

where the maps $m$ indicate the algebra $C$ multiplication acting on a $C$-module.

It then follows that $\mathfrak{P}\left(L_{n}\right)$ is quasi-isomorphic to the $p$-complex

$$
\begin{gathered}
\cdots \stackrel{m}{\longrightarrow} A(n) \otimes I_{3}=A(n) \otimes I_{3} \stackrel{m}{\longrightarrow} A(n) \otimes I_{2} \longrightarrow \\
\qquad A(n) \otimes I_{1}=A(n) \otimes I_{1} \stackrel{m}{\longrightarrow} A(n) \longrightarrow 0 .
\end{gathered}
$$


As the summand decomposition (5.1) is also a decomposition of $p$-DG modules, we have that $A(n)$ contains $x^{n-1} C \cong \mathbb{k}\{2 n-2\}$ as a $p$-complex direct summand. Hence $\mathfrak{P}\left(L_{n}\right)$ contains the infinite $p$-complex (up to an overall shift of $2 n-2$ )

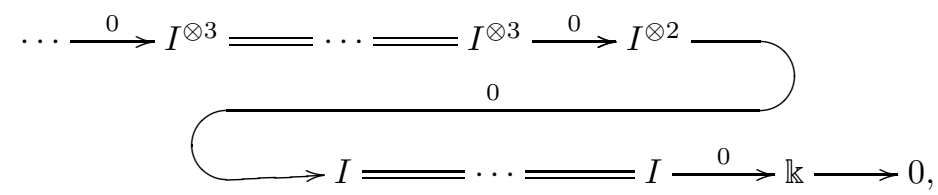

where the zero-maps come from the fact that the element $x^{n-1}$ kills the ideal $I$ : Under the map $m$, we have, for any $y \otimes w-1 \otimes y w \in I_{k}$, where $y \in I$ and $w \in I_{k-1}$,

$$
x^{n-1} \otimes_{C}(y \otimes w-1 \otimes y w)=-x^{n-1} \otimes y w \mapsto-x^{n-1} y w=0 .
$$

The theorem now follows.

5.3. Two examples. In Theorem [5.5, when $n-1$ is not divisible by $p$, the number of copies of $C$ in each piece of the property- $(\mathrm{P})$ replacement of $\mathbb{k}$ grows exponentially. However, there are two special cases where the bar resolution used can be replaced by a much smaller resolution: the number of copies of $C$ in each degree is almost a constant.

ExAmple 5.7. We consider the case when $n=(k+1) p$ for some $k \in \mathbb{N}$. As before, first we take $C_{0}:=C \cdot 1_{0}$ and map it onto $\mathbb{k}$ by identifying $\mathbb{k}$ with the lowest degree subspace spanned by $1_{0}$. Next, we form the filtered complex (c.f. Example 3.5)

$$
C 1_{1} \stackrel{x^{k p+1}}{\longrightarrow} C 1_{0}
$$

Since $\partial^{p-1}\left(x^{k p+1}\right)=0$, this is a rank-two finite cell module.

We compute that

$$
\begin{aligned}
\partial^{p-1}\left(x^{k p+1} 1_{1}\right) & =\sum_{i=0}^{p-1}\left(\begin{array}{c}
p-1 \\
i
\end{array}\right) \partial^{i}\left(x^{k p+1}\right) \partial^{p-i-1}\left(1_{1}\right) \\
& =\sum_{i=0}^{p-2} \frac{(p-1) !}{i !(p-1-i) !} i ! x^{k p+1+i} \partial^{p-i-2}\left(x 1_{0}\right) \\
& =\sum_{i=0}^{p-2} \frac{(p-1) !}{(p-1-i)} x^{(k+1) p} 1_{0} .
\end{aligned}
$$

The second equality holds since $\partial^{p-1}\left(x^{k p+1}\right)=0$. Now, as $i$ ranges over $\{0, \ldots, p-2\}, 1 /(p-1-i)$ ranges over all non-zero elements of $\mathbb{F}_{p}$, therefore the sum in the last term is zero.

It follows that we may repeat the construction, and we obtain an infinite filtered module satisfying property $(\mathrm{P})$ as follows:

$$
\cdots \stackrel{x^{k p+1}}{\longrightarrow} C\{2(r+1) k p\} \stackrel{x^{k p+1}}{\longrightarrow} C\{2 r k p\} \stackrel{x^{k p+1}}{\longrightarrow} \cdots \stackrel{x^{k p+1}}{\longrightarrow} C\{2 k p\} \stackrel{x^{k p+1}}{\longrightarrow} C .
$$

This resolution of $\mathbb{k}$ as a left $p$-DG module has an interesting implication in the Grothendieck group $K_{0}(C)$, which says that

$$
[\mathbb{k}]=\sum_{r=0}^{\infty} q^{2 r k p}[C] .
$$

Since $q^{2 p}=1$ in $\mathbb{O}_{p}$, this equation reduces to

$$
[\mathbb{k}]=\sum_{r=0}^{\infty}[C] .
$$

Although the identity $\sum_{r=0}^{\infty} 1=1 /(1-1)$ no longer makes sense, yet equation (5.10) can be understood as

$$
[C]=[\mathbb{k}]+[I]=[\mathbb{k}]+\sum_{i=1}^{p-1} q^{2 i}[\mathbb{k}]=[\mathbb{k}]-[\mathbb{k}]=0 .
$$


EXAMPLE 5.8. In this example we consider Theorem [5.5] when $n=k p+2(k \geq 0)$, in which case the $p$-DG resolution of $\mathbb{k}$ over $C$ may also be simplified significantly.

For $j=0, \ldots, k-1$ there are contractible $p$-complexes of $C$ of the form

$$
\left(x^{j p+1} \rightarrow x^{j p+2} \rightarrow \cdots \rightarrow x^{(j+1) p}\right) .
$$

Contracting these $p$-complexes one establishes a quasi-isomorphism between $C$ and $\mathbb{k}\left[x^{k p+1}\right] /\left(x^{k p+1}\right)^{2}$ with trivial differential.

As a $p$-DG module over $C$, the trivial module $\mathbb{k}$ is quasi-isomorphic to

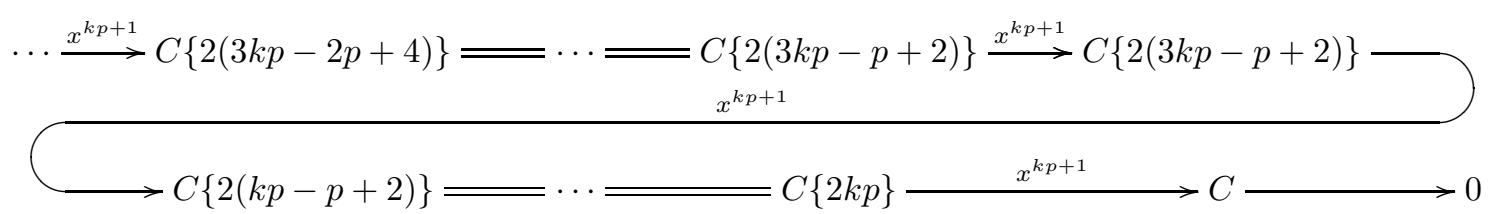

Similar as above, this equation has an interesting Grothendieck group implication saying that

$$
[\mathbb{k}]=\sum_{r=0}^{\infty}(-1)^{r} q^{2 r}[C] .
$$

Although, as before, $q^{2}$ is a root of unity in $\mathbb{O}_{p}$, and it does not make sense for $\sum_{r=0}^{\infty}(-1)^{r} q^{2 r}$ to converge, this equation can, however, be "explained" via the equality of symbols

$$
[C]=[\mathbb{k}]+[I]=[\mathbb{k}]+q^{2(k p+1)}[\mathbb{k}]=\left(1+q^{2}\right)[\mathbb{k}] .
$$

Both examples are instances of why one wants to restrict to compact objects for defining the Grothendieck group of the triangulated category $\mathcal{D}(C)$ of a $p$-DG algebra. The module $\mathbb{k}$ in these cases are not compact in $\mathcal{D}(C)$. We refer the reader to EQ13 for a discussion about compact $p$-DG modules over a positive $p$ - $D G$ algebra. This notion includes both $C$ and $A$ in this paper as special instances.

\section{References}

[AL13] R. Anno and T. Logvinenko, Spherical DG-functors, arXiv:1309.5035

[BCGPM14] C. Blanchet, F. Costantino, N. Geer, and B. Patureau-Mirand, Non semi-simple sl(2) quantum invariants, spin case, arXiv:1405.3490

[BCL14] A. Beliakova, Q. Chen, and T. Lê, On the integrality of Witten-Reshetikhin-Turaev 3-manifold invariants, Quantum Topol. 5 (2014), no. 1, 99-141, arXiv:1010.4750.

[BFK99] J. Bernstein, I. B. Frenkel, and M. Khovanov, A categorification of the Temperley-Lieb algebra and Schur quotients of $U\left(\mathfrak{s l}_{2}\right)$ via projective and Zuckerman functors, Selecta Math. (N.S.) 5 (1999), no. 2, 199-241, arXiv:math/0002087.

[CF94] L. Crane and I. B. Frenkel, Four dimensional topological quantum field theory, Hopf categories, and the canonical bases, J. Math. Phys. 35 (1994), no. 10, 5136-5154, arXiv:hep-th/9405183

[CK12] B. Cooper and V. Krushkal, Categorification of the Jones-Wenzl projectors, Quantum Topol. 3 (2012), no. 2, 139-180, arXiv:1005.5117.

[EKL14] A. P. Ellis, M. Khovanov, and A. D. Lauda, The odd nilHecke algebra and its diagrammatics, Int. Math. Res. Not. IMRN (2014), no. 4, 991-1062, arXiv:1111.1320

[EQ13] B. Elias and Y. Qi, A categorification of some small quantum groups II, arXiv:1302.5478

[EQ15a] A A categorification of quantum sl(2) at prime roots of unity, arXiv:1503.05114

[EQ15b] A. P. Ellis and Y. Qi, The differential graded odd nilHecke algebra, arXiv:1504.01712

[FSS12] I. B. Frenkel, C. Stroppel, and J. Sussan, Categorifying fractional Euler characteristics, Jones-Wenzl projectors and 3j-symbols, Quantum Topol. 3 (2012), no. 2, 181-253, arXiv:1007.4680

[Kap96] M. Kapranov, On the q-analog of homological algebra, arXiv:q-alg/9611005

[Kel06] B. Keller, On differential graded categories, International Congress of Mathematicians II (2006), 151-190, arXiv:math/0601185.

[Kho00] M. Khovanov, A categorification of the Jones polynomial, Duke Math. J. 101 (2000), no. 3, 359-426, arXiv:math/9908171.

[Kho06] Hopfological algebra and categorification at a root of unity: the first steps, arXiv:math/0509083

[KL09] M. Khovanov and A. D. Lauda, A diagrammatic approach to categorification of quantum groups I, Represent. Theory 13 (2009), 309-347, arXiv:0803.4121.

[KL10] _ A categorification of quantum sl(n), Quantum Topol. 2 (2010), no. 1, 1-92, arXiv:0807.3250. 
[KL11] _ A diagrammatic approach to categorification of quantum groups II, Trans. Amer. Math. Soc. 363 (2011), no. 5, 2685-2700, arXiv:0804.2080

[KLMS12] M. Khovanov, A. D. Lauda, M. Mackaay, and M. Stošić, Extended graphical calculus for categorified quantum sl(2), Mem. Amer. Math. Soc., vol. 219, 2012, arXiv:1006.2866

[KQ15] M. Khovanov and Y. Qi, An approach to categorification of some small quantum groups, Quantum Topol. 6 (2015), no. 2, 185-311, arXiv:1208.0616

[KS02] M. Khovanov and P. Seidel, Quivers, Floer cohomology, and braid group actions, J. Amer. Math. Soc. 15 (2002), 203-271, arXiv:math/0006056

[Lau10] A. D. Lauda, A categorification of quantum sl(2), Adv. Math. 225 (2010), no. 6, 3327-3424, arXiv:0803.3652

[May42a] W. Mayer, A new homology theory, Ann. of Math. (2) 43 (1942), no. 2, 370-380.

[May42b] _ A new homology theory. II, Ann. of Math. (2) 43 (1942), no. 3, 594-605.

[Mir15] D. Mirmohades, Categorification of the ring of cyclotomic integers for products of two primes, arXiv:1506.08755

[Qi14] Y. Qi, Hopfological algebra, Compos. Math. 150 (2014), no. 01, 1-45, arXiv:1205.1814.

[QS13] Y. Qi and J. Sussan, A categorification of the Burau representation at prime roots of unity, arXiv:1312.7692

[Rou08] R. Rouquier, 2-Kac-Moody algebras, arXiv:0812.5023.

[Roz14] L. Rozansky, An infinite torus braid yields a categorified Jones-Wenzl projector, Fund. Math. 225 (2014), no. 1, 305-326, arXiv:1005.3266.

[RT90] N. Y. Reshetikhin and V. G. Turaev, Ribbon graphs and their invariants derived from quantum groups, Comm. Math. Phys. 127 (1990), no. 1, 1-26.

[Sar95] K. S. Sarkaria, Combinatorial methods in topology, Available at http://kssarkaria.org/

[SS] C. Stroppel and J. Sussan, A Lie theoretic categorification of the coloured Jones polynomial, In preparation.

[SS14] _ Categorified Jones-Wenzl projectors: a comparison, Perspectives in representation theory, Contemp. Math., vol. 610, Amer. Math. Soc., Providence, RI, 2014, arXiv:1105.3038 pp. 333-351.

[ST01] P. Seidel and R. Thomas, Braid group actions on derived categories of coherent sheaves, Duke Math. J. 108 (2001), no. 1, 37-108, arXiv:math/0001043

[Web15] B. Webster, Knot invariants and higher representation theory, arXiv:1309.3796.

[Wit89] E. Witten, Quantum field theory and the Jones polynomial, Commun. Math. Phys. 121 (1989), no. 3, 351-399.

Department of Mathematics, Yale University, New Haven, CT 06511, USA

E-mail address: you.qi@yale.edu

Department of Mathematics, CUny Medgar Evers, Brooklyn, NY, 11225, USA

E-mail address: jsussan@mec.cuny.edu 